\title{
EDUCATIONAL APPROACHES TO ADDRESS FAKE NEWS - PRELIMINARY INSIGHTS FROM A SYSTEMATIC REVIEW
}

\author{
Christoph Eisemann and Christoph Pimmer \\ FHNW University of Applied Sciences and Arts Northwestern Switzerland \\ Peter Merian-Strasse 86, 4002 Basel-Switzerland
}

\begin{abstract}
Fake and false news, an unfortunate hallmark of today's information society, have serious political and societal consequences. Little systematic knowledge is available about effective learning, teaching and awareness-raising strategies that help users in addressing fake news. This study reports preliminary results from a systematic literature review aimed at systematising different approaches and determining their effectiveness. Three main approaches emerged in the analysis: Firstly, the findings suggest that strategies to correct existing misconceptions caused by fake news have limited effectiveness and can be even counterproductive, particularly for polarising topics. Secondly, the evidence on the effectiveness of training on fake news detection methods is encouraging but inconclusive. Thirdly, despite the common perception that fake news detection needs to be linked to an understanding of the economic, ideological and cultural dimensions of media systems, the few empirical studies found in this area did little to support this claim. A tentative conclusion from these findings is the need to integrate education on false news and training on fake news detection strategies in educational programmes as early as children's media consumption starts.
\end{abstract}

\section{KEYWORDS}

Fake News, False News, Misinformation, Disinformation, Fake News Resilience, Education, Systematic Review

\section{INTRODUCTION}

The circulation of fake and false news has become emblematic for today's information society. To address this issue, Lazer et al. (2018) have called for a multidisciplinary effort in their recent article in the journal Science. This effort should include technical measures aimed at preventing the exposure of individuals to fake news. Secondly, it should also contain measures that empower internet users in dealing with fake news. With the goal of developing a better understanding of the effectiveness of different educational approaches and methods that can support users in tackling fake news, the present study focuses on the second aspect highlighted by Lazer et al. (2018). Despite the considerable number of individual studies about this topic and systematic reviews on media literacy (e.g. Jeong et al., 2012), the literature in this field is still fragmented. With reference to this gap, the following research question was addressed by a systematic literature review: How effective are educational approaches that help users in dealing with fake news?

\section{METHODOLOGY}

The search was conducted in April 2020 and included contributions from 2000-2020 in the databases ERIC, OVID Medline, APA PsycInfo and PsycARTICLES via the platform OVID (using title and abstract search) and in the Web of Science. The search combined the two domains of (1) fake and false news and (2) educational approaches in the following way: (Fake news OR false news OR misinformation OR disinformation) AND (Education OR training OR interventio* OR debunk* OR debias*). Upon deleting 179 duplicates, the two authors screened the abstracts of the remaining 955 articles against the following criteria: (1) educational interventions, i.e. instruction, training, or awareness-raising measures, which were (2) evaluated empirically, 
i.e. through quantitative, qualitative or mixed-methods studies; (3) articles aimed at the change of knowledge, behaviour or attitudes, i.e., excluding cross-sectional surveys or theoretical papers; (4) studies that report outcomes and/or users' experience of these interventions (reports that described technical designs only were excluded); (5) articles with a sound methodological design (including a detailed description of data collection and analyses methods according to established standards). After the independent review of this corpus of studies by the two authors against these criteria and a subsequent consensus discussion, a number of 14 articles was retained and summarised in the present short paper, alongside five articles that were identified through a selective search in Google scholar, resulting in a total 19 studies. In this short paper, we present tentative findings grouped around three emerging themes that were identified through thematic analysis.

\section{FINDINGS}

\subsection{Reactive, Fact-Based Correction of Misinformation}

A relatively large number of studies examined interventions aimed at correcting already internalized misconceptions (Cook et al., 2017; Hameleers and van der Meer, 2020; Murrock et al., 2018; Rich et al., 2017; Nyhan et al., 2014; Paynter et al., 2019; Pluviano et al., 2019, 2017; Tarchi, 2019). Correcting users' misbeliefs and misconceptions is a reactive strategy, which seeks to redress the negative impact of an individual's exposure to prior misinformation and disinformation. Although the provision of information about what is right and wrong in a fact-based manner might seemingly be the most intuitive and straightforward pedagogical approach, the literature suggests that it is linked to limited effectiveness. Some interventions were even counterproductive for some groups, in particular when the topic was polarising, such as vaccination (Nyhan et al., 2014; Paynter et al., 2019; Rich et al., 2017). An example is the study of Nyhan et al. (2014) who compared in their large, nationally-representative, randomised controlled trial four different instructive strategies aimed at reducing vaccine misperceptions. These strategies included the provision of: (1) an explanation of the lack of evidence between vaccines and autism; (2) information about the dangers of missing out on vaccinations; (3) images of children suffering from diseases that could be prevented by vaccines; (4) a dramatic narrative about an infant who almost died of a preventable illness. Whereas some misperceptions were reduced, none of these interventions increased parental intent to vaccinate a future child and even decreased the intent to vaccinate among parents with the least favourable vaccine attitudes (Nyhan et al., 2014).

What makes the correction of misinformation more effective is the combination of several strategies (Paynter et al., 2019), the provision of additional, explanatory information (Paynter et al., 2019; Rich et al., 2017), and the communication of scientific consensus. For example, Cook et al. (2017) found that highlighting scientific consensus on climate change was effective in neutralizing adverse effects of misinformation. This finding is similar to Van der Linden et al.'s (2017) study which showed that the communication of scientific consensus on human-caused climate change significantly increased the public perception of the expert consensus. However, also these approaches need to be treated with caution as some studies found that effects were not sustained over time (Paynter et al., 2019).

\subsection{Proactive Training of Specific Fake News Detection Methods}

The second main approach that emerged in the analysis was to equip users with specific methodological and technical repertoire, i.e. knowledge, skills and tools that can help them in recognising fake news (Basol et al., 2020; Jones-Jang et al., 2019; Katsaounidou et al., 2019; Kim and Dennis, 2019; Lutzke et al., 2019; McGrew, 2020; Murrock et al., 2018; Roozenbeek and van der Linden, 2019; Tsipursky et al., 2018a, 2018b; Velautham, 2017).

Within this pro-active approach, various methods can be differentiated. Making participants aware of guidelines and techniques on fake news detection was reported to be successful in two studies (Murrock et al., 2018, Lutzke et al., 2019). For example, prompting users with questions, for instance on the authorship or style of a Facebook post, decreased their likelihood to trust, like and share fake news - but not legit news - about climate change in comparison with a control group (Lutzke et al., 2019). Results from another study are more ambiguous, because the effect of nudging users to scrutinize the authorship of news articles made them more sceptical of all articles regardless of whether they were true of false (Kim and Dennis, 2019). More extensive 
course formats showed clearer effects: The majority of the participants of a digital literacy programme reported retaining their behaviour of cross-checking news even 1.5 years after the training. Their abilities of recognising fake news declined only slightly (Murrock et al., 2018). Another, more extensive, eight-lesson-course through which students were taught to evaluate digital content found that they demonstrated a significantly improved ability to investigate the source of a website, critique evidence and locate reliable sources during an open internet search (McGrew, 2020).

However, methodological-technical fake news detection training was no guarantee of success. In a large Indian field study, users were trained through an hour-long media literacy intervention. The training, which included the use of reverse image search and fact checkers, did not only show no increase in fake news detection skills; for some partisan groups, it even decreased their abilities to spot fake news (Tan, 2019).

A noteworthy approach is gamification (Basol et al., 2020, Roozenbeek and van der Linden, 2019, Katsaounidou et al., 2019, Urban et al., 2018). One example is a digital game that included the use of tools such as search engine search, reverse image research, image verification assistant and fact checkers (Katsaounidou et al., 2019). The analysis was restricted to self-perceived effects, and more than $60 \%$ of the participants reported that it enhanced their digital literacy (Katsaounidou et al., 2019). An interesting story-telling element was featured in the digital game Bad News, where users learned by assuming the role of fake news disseminators. The large-scale study with $15^{\prime} 000$ participants found that fake news detection abilities increased between the pre-test and post-test (Roozenbeek and van der Linden, 2019). A second study found positive effects also in comparison to a control group (Basol et al., 2020). However, the findings from our own randomised controlled trial suggest that in higher education contexts the game had no meaningful effects (Pimmer et al., 2020, accepted). A tool that is often integrated in educational interventions is fact checkers (Hameleers and van der Meer, 2020, McGrew, 2020, Katsaounidou et al., 2019). Two large-scale experiments found that their use was successful because it decreased participants' agreement with attitudinally congruent political misinformation. However, fact-checkers were most likely used when they confirmed prior attitudes - and avoided when they were incongruent with previous beliefs, which points to a confirmation bias (Hameleers and van der Meer, 2020).

\subsection{Development of a Critical Understanding of Media Systems}

The third main approach identified in this review is the provision of users with knowledge of the wider, political economic, ideological and cultural fabric and the dynamics of media systems and media production with the goal of helping them to critically reflect and contextualise news. However, contrary to scholarly conceptions of the importance of a broader understanding of the overall media system (e.g. Buckingham, 2019), the very few studies identified in this area provided little evidence to substantiate this rationale. One exception that points to the potential linkage between knowledge of the wider media system and concrete fake news detection skills is a study from the Ukraine. Citizen were instructed through a learning programme which included content on the nature of information, manipulation, propaganda, fake news and their dangers, types of mass media and media ownership (Murrock et al., 2018). The curriculum considered also the contextual specificities of the news media environment in the Ukraine. The post-hoc evaluation found that participants in the programme, who were also instructed in fake news detection strategies, had in fact higher levels of knowledge of the news media environment and they were better in distinguishing false from correct news in comparison to a control group, which was not subject of any intervention. Interestingly, across intervention and control group, there was a linkage between fake news detection skills and knowledge about the workings of news media, although the research design did not permit the closer examination of this relationship. In addition, some digital games touch on the broader dynamics through which disinformation is being shared, albeit to a very limited extent (Urban et al., 2018, Roozenbeek and van der Linden, 2019, Basol et al., 2020). It was, in fact, suggested that a way to improve one game would be to integrate more information about underlying political motives and dynamics (Urban et al., 2018). 


\section{CONCLUSION, LIMITATIONS AND NEXT STEPS}

The tentative results of this review show that there is a relatively convincing evidence base suggesting that pre-existing misconceptions can at best be removed with difficulties. This can be explained with the avoidance of cognitive dissonance and the concept of motivated reasoning, with people seeking to confirm what they already believe (Lewandowsky et al., 2012). Secondly, there is encouraging but inconclusive evidence that shows the effectiveness of interventions which train users in applying concrete fake news detection methods. However, some studies reported no or even adverse effects, and studies very rarely measured the mid-term and long-term impact. Moreover, many of these studies were carried out in artificial laboratory settings in which users were aware of their exposure to misinformation. Thirdly, despite the common understanding that fake news detection should be linked to the broader knowledge of the wider media system (Buckingham, 2019), the corpus of studies that focused on this aspect identified in the present review is very thin and inconclusive. The inconclusive nature of this relationship is also reflected by a cross-sectional study in which peoples' abilities to find reliable information online was linked to better fake news detection skills; however, news literacy, i.e. their knowledge about the news production system was not (Jones-Jang et al., 2019). The "missing link" between media system knowledge and fake news detection skills could result from our search strategy (see the limitation section) but also from the methodological challenges linked to measuring these linkages. Finally, an interesting strand to be followed is gamification, where results can be viewed cautiously optimistic. A proposition to be examined more thoroughly in the future could be that the less "authoritarian" and more subtle and playful ways of gamification could be more adequate in changing peoples' perception of and knowledge on fake news.

The tentative findings from this review need to be interpreted with caution. Firstly, because of limitations in the body of studies identified (such as the focus on short term effects), and secondly, due to specific scope of our search strategy, which was systematic but not comprehensive. A limitation was that there might be a potentially interesting corpus of media literacy studies which has already addressed similar concepts without using the terms that we have considered in our search. In the next step, we thus plan to enhance the scope of our search by further scrutinising media literacy and digital literacy studies in connection with fake and false news. However, we would argue that the current findings present some interesting insights. A tentative practical recommendation from this review is to anchor news analysis and fake news detection education as early as possible in education curricula to prevent misconceptions from striking roots and to equip users with the adequate methodological repertoire.

\section{ACKNOWLEDGEMENT}

We would like to thank the dean of BSc Betriebsökonomie Basel and the management board from the School of Business FHNW University of Applied Sciences and Arts Northwestern Switzerland for their support of this study. We are also very grateful for the students who agreed to participate in this research.

\section{REFERENCES}

Basol, M., Roozenbeek, J., van der Linden, S. 2020. Good News about Bad News: Gamified Inoculation Boosts Confidence and Cognitive Immunity Against Fake News. Journal of Cognition, 3.

Beker, K., Kim, J., Van Boekel, M., van den Broek, P., Kendeou, P., 2019. Refutation texts enhance spontaneous transfer of knowledge. Contemp. Educ. Psychol. 56, 67-78. https://doi.org/10.1016/j.cedpsych.2018.11.004

Cook, J., 2019. Understanding and countering misinformation about climate change. Handb. Res. Decept. Fake News Misinformation Online, Advances in media, entertainment, and the arts (AMEA) book series. 281-306. https://doi.org/10.4018/978-1-5225-8535-0.ch016

Hameleers, M., van der Meer, T.G.L.A., 2020. Misinformation and Polarization in a High-Choice Media Environment: How Effective Are Political Fact-Checkers? Commun. Res. 47, 227-250. https://doi.org/10.1177/0093650218819671

Jeong, S.-H., Cho, H., Hwang, Y., 2012. Media literacy interventions: A meta-analytic review. Journal of Communication, $62,454-472$. 
Jones-Jang, S.M., Mortensen, T., Liu, J., 2019. Does Media Literacy Help Identification of Fake News? Information Literacy Helps, but Other Literacies Don’t. Am. Behav. Sci. https://doi.org/10.1177/0002764219869406

Katsaounidou, A., Vrysis, L., Kotsakis, R., Dimoulas, C., Veglis, A., 2019. MAthE the Game: A Serious Game for Education and Training in News Verification. Educ. Sci. 9. Lazer, D. M., Baum, M. A., Benkler, Y., Berinsky, A. J., Greenhill, K. M., Menczer, F., Metzger, M. J., Nyhan, B., Pennycook, G., Rothschild, D., 2018. The science of fake news. Science, 359, 1094-1096.

Kim, A., Dennis, A. R., 2019. Says who? The effects of presentation format and source rating on fake news in social media. Mis Quarterly, 43(3), 1025-1039.

Lewandowsky, S., Ecker, U.K.H., Seifert, C.M., Schwarz, N., Cook, J., 2012. Misinformation and its correction: Continued influence and successful debiasing. Psychol. Sci. Public Interest 13, 106-131. https://doi.org/10.1177/1529100612451018

Linden, S. van der, Leiserowitz, A., Rosenthal, S., Maibach, E., 2017. Inoculating the Public against Misinformation about Climate Change. Glob. Chall. 1, 1600008. https://doi.org/10.1002/gch2.201600008

Lutzke, L., Drummond, C., Slovic, P., Arvai, J., 2019. Priming critical thinking: Simple interventions limit the influence of fake news about climate change on Facebook. Glob. Environ. CHANGE-Hum. POLICY Dimens. 58. https://doi.org/10.1016/j.gloenvcha.2019.101964

McGrew, S., 2020. Learning to evaluate: An intervention in civic online reasoning. Comput. Educ. 145. https://doi.org/10.1016/j.compedu.2019.103711

Murrock, E., Amulya, J., Druckman, M., Liubyva, T., 2018. Winning the War on State-Sponsored Propaganda: Results from an Impact Study of a Ukrainian News Media and Information Literacy Program. J. Media Lit. Educ. 10, 53-85.

Nyhan, B., Reifler, J., Richey, S., Freed, G. L., 2014. Effective Messages in Vaccine Promotion: A Randomized Trial. Pediatrics, 133, E835-E842.

Paynter, J., Luskin-Saxby, S., Keen, D., Fordyce, K., Frost, G., Imms, C., Miller, S., Trembath, D., Tucker, M., Ecker, U., 2019. Evaluation of a template for countering misinformation-Real-world autism treatment myth debunking. PLoS ONE 14. (1): e0210746. https://doi.org/10.1371/journal.pone.0210746

Pimmer, C., Eisemann, C., \& Mateescu, M. (2020 (accepted)). Fake news resilience through online games? Tentative findings from a randomized controlled trial in higher education. Paper to be presented at the CELDA - 17th International Conference on Cognition and Exploratory Learning in Digital Age 2020, Lisbon, Portugal.

Rich, P.R., Van Loon, M.H., Dunlosky, J., Zaragoza, M.S., 2017. Belief in Corrective Feedback for Common Misconceptions: Implications for Knowledge Revision. J. Exp. Psychol.-Learn. Mem. Cogn. 43, $492-501$. https://doi.org/10.1037/xlm0000322

Roozenbeek, J., van der Linden, S., 2019. Fake news game confers psychological resistance against online misinformation. PALGRAVE Commun. 5. https://doi.org/10.1057/s41599-019-0279-9

Tarchi, C., 2019. Identifying fake news through trustworthiness judgements of documents. Cultura Y Educacion, 31(2), 369-406. doi:10.1080/11356405.2019.1597442

Tsipursky, G., Votta, F., Mulick, J.A., 2018. A psychological approach to promoting truth in politics: The Pro-Truth Pledge. J. Soc. Polit. Psychol. 6, 271-290. https://doi.org/10.5964/jspp.v6i2.856

Urban A., Hewitt, C., Moore, J., 2018. Fake it to make it, media literacy, and persuasive design: Using the functional triad as a tool for investigating persuasive elements in a fake news simulator. Proceedings of the Association for Information Science and Technology, 55, 915-916.

Velautham, L., 2017. Designing an Intervention to Promote Critical Thinking about Statistics in the General Public. Berkeley Rev. Educ. 7, 113-119. 